\title{
Thoughts on galactic magnetism *
}

\author{
Kandaswamy Subramanian \\ National Centre for Radio Astrophysics, \\ Tata Institute of Fundamental Research, \\ Poona University Campus, Ganeshkind, \\ Pune 411007, India
}

\begin{abstract}
Magnetic fields correlated on several kiloparsec scales are seen in spiral galaxies. Their origin could be due to amplification of a small seed field by a turbulent galactic dynamo. We critically review the current status of the galactic dynamo, especially some of its problems and possible solutions. We also comment on the nature of seed magnetic fields, needed to prime the dynamo.
\end{abstract}

\section{INTRODUCTION}

Magnetic fields in spiral galaxies have strengths of order few $10^{-6} G$, and are coherent on scales of several kpc [i]. In several disk galaxies, like M51 and NGC 6946, they are also highly correlated (or anti-correlated) with the optical spiral arms. How do such ordered, large-scale fields arise? One possibility is dynamo amplification of a weak but nonzero seed field $\sim 10^{-19}-10^{-23} G$, provided the galactic dynamo can operate efficiently to exponentiate the field by a factor $\sim 30-40$. We critically review here some of the issues relevant to the operation of the galactic dynamo, the problems which arise and possible solutions. We also touch upon the origin of the seed magnetic field, needed for dynamo amplification.

The evolution of the magnetic field, in the MHD approximation, is described by the induction equation

$$
\frac{\partial \mathbf{B}}{\partial t}=\nabla \times(\mathbf{v} \times \mathbf{B}-\eta \nabla \times \mathbf{B})
$$

provided one assumes the usual form of Ohms law and neglects the displacement current term in Maxwells equation. Here $\mathbf{B}$ is the magnetic field, $\mathbf{v}$ the velocity of the fluid and $\eta$ the resistivity. If $\eta \rightarrow 0$ the magnetic flux through any area in the fluid is conserved during the motion of the fluid. The presence of a finite resistivity allows for a violation of such 'flux freezing' and the magnetic Reynolds number (MRN) $R_{m}=v L / \eta$ measures the relative importance of flux freezing versus resistive diffusion. Here $v$ and $L$ are typical velocity and length scales of the fluid motions. In most astrophysical contexts flux freezing greatly dominates over diffusion with $R_{m}>>1$.

$\mathbf{B}=0$ is a perfectly valid solution of the induction equation. So there would be no magnetic field generated if one were to start with a zero magnetic field. The universe probably did not start with an initial magnetic field. One therefore needs some way of violating the induction equation and produce a cosmic battery effect, to drive curents from a state with initially no current. There are a number of such battery mechanisms which have been suggested [2]. All of them lead to only small fields, much smaller than the galactic fields. Therefore dynamo action, due to a velocity field acting to exponentiate small seed fields efficiently, is needed to explain observed fields. We first briefly comment on a cosmic battery before discussing dynamos in detail.

\section{COSMIC BATTERIES AND SEED FIELDS FOR THE DYNAMO}

The basic problem any battery has to address is how to produce finite currents from zero currents? Most astrophysical mechanisms use the fact that positively and negatively charged particles in a charge-neutral universe, do not have identical properties. For example if one considered a gas of ionised hydrogen, then the electrons have a much smaller mass compared to protons. This means that for a given pressure gradient of the gas the electrons tend to be accelerated much more than the ions. This leads in general to an electric field, which couples back positive and negative charges, of the form $\mathbf{E}_{T}=-\nabla p_{e} / e n_{e}$, where $p_{e}$ and $n_{e}$ are the electron pressure and number density, respectively. If such a thermally generated electric field has a curl, then by Faradays law of induction a magnetic field can grow. Taking $p_{e}=n_{e} k T$ with $T$ the electron temperature we have $\nabla \times \mathbf{E}_{T}=(c k / e)\left(\nabla n_{e} / n_{e}\right) \times \nabla T$. So $\mathbf{E}_{T}$ has a

\footnotetext{
* It is a pleasure to dedicate this article to Prof. Jayant Narlikar, my first research Guru, though he may want to have little to do with toroidal and poloidal fields and the infamous 'pomega' !
} 
curl only if the density and temperature gradients, are not parallel to each other. The resulting battery effect, known as the Biermann battery, was first proposed as a mechanism for thermal generation of stellar magnetic fields [3].

The Biermann battery can also lead to the thermal generation of seed fields in cosmic ionisation fronts 伍. These ionisation fronts are produced when the first ultra violet photon sources, like quasars, turn on to ionise the intergalactic medium (IGM). The temperature gradient in a cosmic ionisation front is normal to the front. However, a component to the density gradient can arise in a different direction, if the ionisation front is sweeping across arbitrarily laid down density fluctuations, associated with protogalaxies/clusters since these in general have no correlation to the source of the ionising photons. The resulting thermally generated electric field has a curl, and magnetic fields on galactic scales can grow. They turn out to have a strength $B \sim 3 \times 10^{-20} G$. This field by itself is far short of the observed microgauss strength fields in galaxies, but it can provide a seed field, coherent on galactic scales, for a dynamo. Indeed the whole of the IGM is seeded with small magnetic fields. This seed field may infact have the right symmetry properties for the galactic dynamo modes [5]. The Biermann battery has also been invoked to generate seed magnetic fields in galactic or proto-galactic environments 6 .

Larger seed magnetic fields than above can arise if we combine some form of dynamo action with the battery effect. For example, if stellar dynamos work efficiently, and some stars blow out as supernovae, then they can seed the interstellar medium, with significant magnetic fields. Alternatively galactic turbulence can itself lead to a small-scale dynamo (see below) and provide a larger seed for the large-scale galactic dynamo. There have also been attempts to invoke exotic physics in the early universe to produce primordial magnetic fields [2]. (Infact a primordial field in the IGM, which redshifts to a present day value of $\sim 10^{-9} G$, and is correlated on Mpc scales, can significantly perturb structure formation, and cause detectable anisotropies in the cosmic microwave background radiation [7].) It is fair to say at present that most seed field generation mechanisms fall far short of producing large-scale correlated fields at the micro-gauss level. One does need some form of large-scale dynamo action.

\section{THE LARGE-SCALE GALACTIC DYNAMO}

Disk galaxies are differentially rotating systems. Also the magnetic flux is to a large extent frozen into the fluid. So any radial component of the magnetic field will be efficiently wound up and amplified to produce a toroidal component. But this results in only a linear amplification of the field. To obtain the observed galactic fields starting from small seed fields one should find a way to generate the radial component from the toroidal one. If this can be done, the field can grow exponentially and one has a dynamo.

A mechanism to produce the radial field from the toroidal field was invented by Parker [8]. The essential feature is to invoke the effects of cyclonic turbulence in the galactic gas. The interstellar medium (ISM) is assumed to be turbulent, due to for example the effect of supernovae randomly going off in different regions. In a rotating, stratified (in density and pressure) medium like a disk galaxy, such turbulence becomes cyclonic and acquires a net helicity. Helical motions of the galactic gas perpendicular to the disk can draw out the toroidal field into a loop which looks like a twisted $\Omega$. Such a loop is connected to a current and because of the twist this current has a component parallel to the original field. If the motions have a non-zero net helicity, then the random current components parallel to the field, add up coherently. A toroidal current can then result from the toroidal field. Hence, poloidal fields can be generated from toroidal ones.

In quantitative terms, suppose the velocity field is the sum of a mean, large-scale velocity $\mathbf{v}_{0}$ and a turbulent, stochastic velocity $\mathbf{v}_{T}$. The induction equation becomes a stochastic partial differential equation. The equation for various moments of $\mathbf{B}$, can be derived in two ideal limits. First when $R_{m}<<1$, and the distortions to the mean magnetic field are small, and second when $R_{m}>>1$, but the turbulence is assumed to have a delta function (or very small) correlation in time. For galaxies the latter idealisation may be more relevant.

Let us split the magnetic field $\mathbf{B}=\mathbf{B}_{0}+\delta \mathbf{B}$, into a mean field $\mathbf{B}_{0}$ and a fluctuating component $\delta \mathbf{B}$. Here the mean is defined either as a spatial average over scales larger than the turbulent eddy scales or more correctly as an ensemble average. Assume the turbulence to be isotropic, homogeneous and helical. The action of the turbulent velocity field $\mathbf{v}_{T}$, on the magnetic field, the $(\mathbf{v} \times \mathbf{B})$ term, then leads to an extra contribution to the mean electric field of the form $-c \mathbf{E}_{0}=\alpha \mathbf{B}_{0}-\eta_{t} \nabla \times \mathbf{B}_{0}$. Here $\alpha=-(1 / 3) \int<\mathbf{v}_{T}(t) .\left(\nabla \times \mathbf{v}_{T}(s)\right)>d s$, depends on the helical part of the turbulent velocity correlation function, and $\eta_{t}=(1 / 3) \int\left\langle\mathbf{v}_{T}(t) \cdot \mathbf{v}_{T}(s)\right\rangle d s$, called the turbulent diffusion depends on the non-helical part of the turbulence. Here the angular brackets $<>$ denote an ensemble average, over the stochastic velocity field. The induction equation for the mean field, with the extra turbulent component of the mean electric field, is then given by

$$
\frac{\partial \mathbf{B}_{0}}{\partial t}=\nabla \times\left(\mathbf{v}_{0} \times \mathbf{B}_{0}+\alpha \mathbf{B}_{0}-\left(\eta+\eta_{T}\right) \nabla \times \mathbf{B}_{0}\right) .
$$


This kinematic mean-field dynamo equation, can have exponentially growing solutions, which have been studied extensively in the literature [1]. While the $\alpha$-effect is crucial for regeneration of poloidal from toroidal fields, the turbulent diffusion turns out to be also essential for allowing changes in the mean field flux. Also in galaxies, differential rotation (the $\Omega$ effect) is dominant in producing toroidal from radial fields. The growth rates of the galactic ' $\alpha-\Omega$ dynamo', are typically a few times the rotation time scales, of order $10^{9} \mathrm{yr}$. Modulations of $\alpha$, and $\eta_{T}$, due to enhanced turbulence along spiral arms, can also lead to bi-symmetric large-scale fields, correlated with the optical spirals [9].

Note that the mean field has a scale limited only by the size of the system, which can be much bigger than the scales associated with the turbulence. In this sense one has created order from chaos. One may be tempted to refer to this as an inverse cascade, a term which would suggest transfer of power from smaller to larger and larger scales. But reality is more subtle. All scales larger than the turbulent eddy scale can grow simultaneously due to the $\alpha$-effect; but with larger scales growing slower. So the effect can be thought better as a long range interaction between the turbulent scales, and all larger scales.

A physics comment is in order at this stage. When one considers the effect of turbulent fluid motions on a scalar field, like say smoke, one only gets a mean diffusion of smoke, associated with the random walking nature of turbulent fluid motions. But for 'frozen' magnetic fields the induction equation has terms which not only imply a body transport due to the random motions (v. $\nabla \mathbf{B})$, but also a term, B. $\nabla \mathbf{v}$, which describes the generation of magnetic fields due to velocity shear. It is this qualitative difference between magnetic fields and smoke that leads to an alpha effect (provided also that the motions have a non-zero average helicity), over and above turbulent diffusion. As we see below, it also leads to the small-scale dynamo. Note that both $\alpha$ and $\eta_{T}$, depend crucially on the diffusive (random-walk) property of fluid motion. So if due to some reason (see below) the fluid motion becomes wavelike, then the above integrals average to zero, and the alpha effect and turbulent diffusion will be suppressed.

In deriving the mean-field equation, the turbulent velocities have been assumed to be given, and unaffected by the Lorentz forces due to the magnetic field; at least not until the mean large-scale field builds up sufficiently. However this does not turn out to be valid due to the more rapid build up of magnetic noise compared to the mean field, a problem to which we now turn.

\section{THE SMALL-SCALE DYNAMO AND MAGNETIC NOISE}

The problem with the kinematic mean field dynamo is that it is a myth. This is because, the same turbulence which contributes to the $\alpha$-effect, the turbulent diffusion, and associated growth of mean fields, also leads to a more rapid growth of small-scale fields.

In incompressible turbulence, fluid particles random-walk away from each other. This leads to stretching of the field lines attached to these particles, and an exponential increase of field strength. The stretching will also be accompanied by the field being squeezed into smaller and smaller volumes. Suppose one considers a flux tube, of length $l_{t}$, cross section $A$, density $\rho$ and magnetic field $B$. Then flux freezing implies $B A=$ constant, mass conservation $\rho A l_{t}=$ constant, and incompressibility $\rho=$ constant. So as $l_{t}$ increases due to random stretching, the the magnetic field $B \propto l_{t}$ increases and the cross-section $A \propto l_{t}^{-1}$ decreases.

If for the moment one ignores Lorentz forces, then the sqeezing into small volumes, stops only when diffusive scales are reached. Typically the field can be thought of as being in flux ropes, curved on the eddy scale, say $L$, and a thickness of order the diffusive scale, say $r_{d}$ (assuming only a single scale eddy is present). At this stage the energy input into the magnetic field due to random stretching would be comparable to the energy loss in diffusion. This gives $v B / L \sim \eta B / r_{d}^{2}$, implying $r_{d} \sim L / R_{m}^{1 / 2}$. What happens further (whether growth or diffusion wins out), can only be decided by a more quantitative treatment of the problem.

A rigorous analysis of small-scale field dynamics, was first worked out by Kazantsev [10], and elaborated extensively by many authors [11], for the simple case, where the turbulence was assumed to have a delta function correlation in time. We shall mainly draw upon our own work in Ref. [12]. It turns out that, the small-scale dynamo (SSD) can operate under fairly weak conditions; that the MRN associated with the turbulent motions be greater than a critical value $R_{c} \sim 100$. In particular the fluctuating field tangled on a scale $l$, can grow on the turn over time scale of a turbulent eddy of scale $l$, with a growth rate $\Gamma_{l} \sim v_{l} / l$, provided the MRN on that scale $R_{m}(l)=v_{l} l / \eta>R_{c}$. Here $v_{l}$ is the velocity associated with eddies of scale $l$. For Kolmogorov turbulence, since $v_{l} \propto l^{1 / 3}$, the growth rate $\Gamma_{l} \propto l^{-2 / 3}$, and so increases with decreasing $l$. For galactic gas, with a significant neutral component, typically, even the eddies at the cut-off scale of the turbulence, say $l_{c}$, have $R_{m}\left(l_{c}\right)>>R_{c}$.

The spatial structure of the small-scale dynamo generated field is also of great interest. For this it is useful look at the behaviour of the magnetic correlation function, say $w(r, t)=\langle\delta \mathbf{B}(\mathbf{x}, t) . \delta \mathbf{B}(\mathbf{y}, t)\rangle$, where $r=|\mathbf{x}-\mathbf{y}|$. Here the averaging indicated by $\langle>$, is a double ensemble average over both the stochastic velocity and fluctuating magnetic 
fields. From $\nabla \cdot \delta \mathbf{B}=0$, one can show that the curve $r^{2} w(r)$ should enclose zero area. Since $w(0)$ is neccessarily positive, as one goes to larger $r$, there must be some $r \sim d$ say, when $w(r)$ becomes negative. For such $r$, the fluctuating field at the origin, and at a separation $d$ are pointing in 'opposite' directions on average. This can be intepreted as saying that the field lines on average are curved on scale $d$.

For Kolmogorov type turbulence, and if $R_{m}\left(l_{c}\right)>>R_{c}$, the fastest growing mode, has $w(r, t)$ strongly peaked within the diffusive scale of the cut-off scale eddies, $r=r_{d}\left(l_{c}\right)=l_{c} / R_{m}^{1 / 2}\left(l_{c}\right)$, changing sign at $r \sim l_{c}$, and rapidly decaying for larger $r / l_{c}$. One can interpret such a correlation function as implying that field is concentrated into ropes of thickness $r_{d}\left(l_{c}\right)$ and curved on scales of order $l_{c}$. For slower growing modes, with growth rate $\Gamma_{l} \sim v_{l} / l, w(r)$ extends upto $r \sim l$, after which it decays exponentially. For these modes, the small-scale field can be thought of as being concentrated in rope-like structures with thickness of order the diffusive scale $r_{d}\left(l_{c}\right)$ and curved on a scale upto $\sim l$. In general, at the kinematic stage, the growth rate of irreducible higher order correlations, increase with order, indicating that the field becomes highly intermittent in space.

Note that the small-scale dynamo due to even the eddy at the outer scale of galactic turbulence, will lead to the exponetial growth of small-scale fields on a time $\tau=L / v \sim 10^{7}$ yr. (Here we have taken $L \sim 100 \mathrm{pc}$, and a velocity scale $v \sim 10 \mathrm{~km} \mathrm{~s}^{-1}$.) This time is much shorter than the time scale $\sim 10^{9} \mathrm{yr}$ for mean field growth. The magnetic field is then rapidly dominated by the fluctuating component, before the mean field has grown appreciably. If the energy in the small-scale component grows to equipartition with the turbulent energy density, the turbulence could become more wavelike 'Alfvén' turbulence, than an eddy like fluid turbulence. So diffusive effects like the $\alpha$ and $\eta_{T}$, would get suppressed, and mean field growth stopped. How does the galaxy escape this predicament?

\section{SATURATION OF THE SMALL-SCALE DYNAMO}

To answer this question, it is crucial to find out how the small-scale dynamo saturates. We have concentrated on the possibility that the small scale field continues to be intermittent in space, when it saturates, as it was in the kinematic regime. That it saturates as a 'can of worms'; with peak fields being limited by non-linear effects to values of order or slightly larger than equipartition fields, but with most of the space having much smaller fields. Then the average energy density of the saturated small-scale dynamo generated field, may still be sub-equipartition, since it does not fill the volume. And the turbulence will remain eddy like, and preserve diffusive effects like $\alpha$ and $\eta_{t}$. We have given one explicit realisation of the above idea, in Ref. 12, in the case of a galaxy dominated by neutral particles.

In partially ionised plasma, the Lorentz force acts on ions, which are only coupled to neutrals through collisions. This leads to a 'ambipolar' drift of ions (and hence the field) with respect to neutrals. With $\mathbf{v}$ in Eq.(1) replaced by the neutral fluid velocity, the effective diffusivity changes to $\eta_{\text {eff }}=\eta+<\delta \mathbf{B}^{2}>/\left(6 \pi \rho_{i} \nu_{i n}\right)$. Here $\rho_{i}$ is the ion density and $\nu_{i n}$ is the neutral-ion collision frequency. So, as the energy density in the fluctuating field increases, the effective magnetic Reynolds number, for fluid motion on any scale of the turbulence say $R_{a m b i}(l)=v_{l} l / \eta_{e f f}$, decreases. If $R_{a m b i}$ could decrease to $R_{c}$, this itself will lead to a SSD saturation, but for conditions appropriate to galactic gas, $R_{a m b i}$ remains much larger than $R_{c}$. So as the the small-scale field grows in strength, it continues to be concentrated into thin ropy structures, as in the kinematic regime. These flux ropes are curved on the turbulent eddy scales, while their thickness is now set by the diffusive scale determined by the effective ambipolar diffusion.

Other restraining effects have to then limit the SSD. The first of these is due to the growing magnetic tension associated with the curved flux ropes. It acts to straighten out a flux rope, at a rate determined by equating the tension force and frictional drag. Frictional drag also damps the magnetic energy associated with the wrinkle in the rope. Further, small-scale flux loops can collapse and disappear, causing an irreversible sink of magnetic energy into heat. These non-local effects operate on the eddy turnover time scale, when the peak field in a flux rope, say $B_{p}$, has grown to a few times the equipartion value. Their net effect is to make the random stretching needed for the SSD inefficient and hence saturate the SSD. As the field is in flux ropes which do not fill the volume. the average energy density in the saturated small-scale field is still sub-equipartition, and $\alpha$ and $\eta_{T}$ are preserved.

Note that $B_{p}$ has to grow to a larger and larger value, thinner the flux rope, for inefficient random stretching to operate. This is because tension is a volume force $\left(\propto r_{d}^{2} l_{t}\right)$ while drag acts on the surface of the rope and is $\propto r_{d} l_{t}$. But $B_{p}$ cannot grow larger than $\left(8 \pi P_{e x t}\right)^{1 / 2}$, where $P_{e x t}$ is the total pressure in the ISM. At the same time the thickness of flux ropes is larger, greater the ambipolar diffusion, or smaller the ion-density. So the SSD saturates as above, for a given $P_{\text {ext }}$, provided $n_{i}$ is less than a crictical value $n_{i}^{c}$. In the ISM, if $P_{\text {ext }}$ is a factor $F$ greater than the gas pressure (the gas assumed to have $T \sim 10^{4} \mathrm{~K}$ and a density $\left.n_{n}\right)$, one gets $n_{i}^{c} \sim 10^{-2} \mathrm{~cm}^{-3}\left(v / 10 \mathrm{kms}^{-1}\right)^{-3}\left(n_{n} / \mathrm{cm}^{-3}\right)^{2 / 3}(F / 2)^{7 / 3}$. So for a range of 'galactic' like parameters, this picture of small-scale dynamo saturation works.

For larger ion densities $n_{i}>n_{i}^{c}$, the way the SSD saturates is not very clear. The peak field is still limited by the external pressure. But how the flux ropes behave in the post-kinematic stage is yet to be rigorously worked out. It is possible that, when one starts with weak fields, the field is first squeezed into small volumes until limited by magnetic 
pressure. Subsequently, constructive folding of the field, may lead to fusing and thickening of the flux rope, while destructive folding may lead to reconnection, and dissipation. A phenomenological model [13] which incorporates this thickening of flux ropes as the field builds up, drives the SSD into saturation, when the rope thickness becomes of order $L / R_{c}^{1 / 2}$, the peak field reaches equipartition levels, but with the average energy density of order $R_{c}^{-1}$ of equipartition.

Numerical simulations of dynamo action due to mirror-symmetric turbulence [14] or convection [15] have also hinted at a saturated state of SSD as described above; a magnetic field concentrated into flux ropes, occupying a small fraction of the fluid volume, having peak fields comparable or in excess of equipartition value but average magnetic energy density only about $10 \%$ of the kinetic energy density. Such simulations are however limited by the MRN they can achieve. There have also been MHD simulations of the SSD in fourier space, adopting some form of closure approximation, like the EDQNM approximation [16], or the DIA [17]. They have also indicated that the small-scale field could saturate at sub-equipartition levels. However, why this happens and the relation of the fourier space, to the real space calculations which we have emphasised, is not at present clear.

There ofcourse remains the possibility that the SSD in a fully ionised gas saturates only when the energy density in the noise grows comparable to that of the turbulence. However, the spatial structure of the small-scale field, is still likely to be highly intermittent. The large-scale dynamo action will then depend on how such a field responds to turbulent motions, especially whether the field can reconnect efficiently [18]. If reconnection is efficient, then it may allow diffusive transport to still occur, through the forest of small-scale fields, rather like Tarzan, swinging from one rope to another crosses the jungle! Reconnection is an important issue, which deserves much more discussion than we have given (cf. [19]). Another important issue which is just beginning to be addressed [20], is the calculation of $\alpha$ (or $\eta_{T}$ ) in the presence of significant small-scale fields.

In summary, the survival of the diffusive effects needed for large-scale dynamo action could depend crucially on whether the SSD generated fields can saturate at sub-equipartition levels. We feel that this may indeed be possible, if the noise saturates as a 'can of worms'. Note the SSD generated field is indeed spatially intermittent in the kinematic regime. So when one starts from weak fields, kinematic evolution operates for some time, and the small-scale field is driven to an intermittent state. The important question is to what extent it remains so in the non-linear regime, when it saturates. This SSD generated field will also provide a strong seed for the large-scale dynamo [21]. Indeed, in a unified treatment of small and large-scale dynamos, large-scale correlations are produced, from small-scale fields in a way analogous to 'quantum mechanical tunnelling', of the stationary state of the SSD [13]. The helical, turbulent, $\alpha-\Omega$ dynamo still seems to be the best bet for explaining large-scale galactic fields. Ultimately the galactic dynamo is a non-linear dynamo; but a discussion of how it operates in the final saturated regime needs more thought. Our thoughts on galactic magnetism have yielded interesting results but much remains to be done.

[1] Beck, R., Brandenburg, A., Moss, D., Shukurov, A. and Sokoloff, D., 1996, Ann. Rev. Astr. Astrophys. 34, 155. Ruzmaikin, A. A., Shukurov, A. M. \& Sokoloff, D. D., 1988. Magnetic Fields of Galaxies, Kluwer, Dordrecht.

[2] Rees, M.J., 1994. Cosmical Magnetism, ed. Lynden-Bell, D., Kluwer, London, p155; Subramanian, K. 1995, Bull. Astr.Soc. India., 23, 481; Ratra, B., 1992, ApJ Lett., 391, L1; and references therein.

[3] Biermann, L., 1950. Zs. Naturforsch. A., 5, 65; Mestel, L. \& Roxburgh, I.W., 1962. ApJ., $136,615$.

[4] Subramanian, K., Narasimha, D. \& Chitre, S.M., 1994. MNRAS. 271, L15.

[5] Krause, F. \& Beck, R., 1998, A\&A., 335, 789.

[6] Lazarian, A., 1992, A\&A, 264, 326; Kulsrud R. M., Cen, R., Ostriker, J. P., Ryu, D., 1997, ApJ, $480,481$.

[7] Wasserman, I., 1978, ApJ, 224, 337; Subramanian, K. \& Barrow, J. D., 1998, Phys. Rev. D, 58, 083502; Phys. Rev. Lett., $81,3575$.

[8] Parker, E.N., 1955. ApJ., 122, 293; Steenbeck, M, Krause, F. \& Radler, K-H., Z. Naturforsch., 21 a, 369.

[9] Mestel, L. \& Subramanian, K. 1991, MNRAS, 248, 677; Subramanian, K. \& Mestel, L., 1993, MNRAS, 265, 649; Moss, D, 1996, A\&A, 308, 381.

[10] Kazantsev, A. P., 1968, Sov. Phys. - JETP, 26, 1031.

[11] Zeldovich, Ya.B., Ruzmaikin,A.A. \& Sokoloff,D.D., 1983. Magnetic fields in Astrophysics, Gordon and Breach, New York. Vainshtein, S. \& Kichatinov, L. L., 1986, J. Fluid. Mech., 168, 73. Kulsrud, R.M. \& Anderson, S.W., 1992. ApJ., 396, 606.

[12] Subramanian, K. 1998, MNRAS, 294, 718; 1997, preprint, astro-ph/9708216.

[13] Subramanian, K, 1999 (paper in preparation).

[14] Meneguzzi, M., Frisch, U. \& Pouquet, A., 1981. Phys. Rev. Lett., 47, 1060.

[15] Brandenburg, A., Jennings, R. L., Nordlund, A., Rieutord, M., stein, R. F. \& Tuominen, I., 1996. JFM, $306,325$. 
[16] Pouquet, A., Frish, U. \& Leorat, J., 1976, JFM, 77, 321.

[17] Chandran, B. D. G., 1997, ApJ, 482, 156; ApJ, 485, 148.

[18] Vishniac, E. T. 1995, ApJ, 446, 724; Blackman, E., 1996. Phys. Rev. Lett., 77, 2694.

[19] Lazarian, A. \& Vishniac, E. T. preprint, astro-ph/9811037

[20] Field, G., Blackman, E. \& Chou, H., 1999. ApJ (in press)

[21] Beck, R., Poezd, A.D., Shukurov, A. \& Sokoloff, D., 1994.. A \& A, 289, 94. 